\title{
Corrigendum to "All-Atom Four-Body Knowledge-Based Statistical Potentials to Distinguish Native Protein Structures from Nonnative Folds"
}

\author{
Majid Masso (D) \\ School of Systems Biology, George Mason University, 10900 University Blvd. MS 5B3, Manassas, VA 20110, USA \\ Correspondence should be addressed to Majid Masso; mmasso@gmu.edu \\ Received 7 December 2017; Accepted 12 December 2017; Published 8 January 2018 \\ Copyright (C) 2018 Majid Masso. This is an open access article distributed under the Creative Commons Attribution License, which \\ permits unrestricted use, distribution, and reproduction in any medium, provided the original work is properly cited.
}

In the article titled "All-Atom Four-Body Knowledge-Based Statistical Potentials to Distinguish Native Protein Structures from Nonnative Folds" [1], there were errors in the energy values in the last column of Table 8 . The corrected table is shown below.

TABLE 8: All-atom four-body statistical potential derived using a 6-letter alphabet and a $12 \AA$ cutoff.

\begin{tabular}{lcccc}
\hline Quad & Count & $f_{i j k l}$ & $p_{i j k l}$ & $s_{i j k l}$ \\
\hline CCCC & 4107297 & 0.112818 & 0.160748 & 0.15377 \\
CCCM & 1924 & $5.28 E-05$ & 0.00045 & 0.93026 \\
CCCN & 4142684 & 0.11379 & 0.172495 & 0.18067 \\
CCCO & 6462239 & 0.177503 & 0.193701 & 0.03793 \\
CCCS & 297980 & 0.008185 & 0.005072 & -0.207795 \\
CCCX & 2996 & $8.23 E-05$ & 0.000765 & 0.96834 \\
CCMM & 157 & $4.31 E-06$ & $4.73 E-07$ & -0.96026 \\
CCMN & 3758 & 0.000103 & 0.000362 & 0.5452 \\
CCMO & 6511 & 0.000179 & 0.000407 & 0.35687 \\
CCMS & 2320 & $6.37 E-05$ & $1.07 E-05$ & -0.776892 \\
CCMX & 15 & $4.12 E-07$ & $1.61 E-06$ & 0.591 \\
CCNN & 1871781 & 0.051413 & 0.069412 & 0.13036 \\
CCNO & 8544461 & 0.234696 & 0.155892 & -0.177683 \\
CCNS & 128008 & 0.003516 & 0.004082 & 0.06485 \\
CCNX & 2159 & $5.93 E-05$ & 0.000616 & 1.01632 \\
CCOO & 3686844 & 0.101269 & -0.087528 & -0.063328 \\
CCOS & 205846 & 0.005654 & 0.004584 & 0.091103 \\
CCOX & 4995 & 0.000137 & 0.000691 & -0.849914 \\
CCSS & 15467 & 0.000425 & $6.00 E-05$ & 0.64875 \\
CCSX & 148 & $4.07 E-06$ & $1.81 E-05$ & \\
\hline
\end{tabular}


TABLE 8: Continued.

\begin{tabular}{|c|c|c|c|c|}
\hline Quad & Count & $f_{i j k l}$ & $p_{i j k l}$ & $s_{i j k l}$ \\
\hline CCXX & 161 & $4.42 E-06$ & $1.37 E-06$ & -0.510349 \\
\hline CMMM & 29 & $7.97 E-07$ & $2.21 E-10$ & -3.557768 \\
\hline CMMN & 164 & $4.50 E-06$ & $2.54 E-07$ & -1.249604 \\
\hline CMMO & 293 & $8.05 E-06$ & $2.85 E-07$ & -1.451272 \\
\hline CMMS & 665 & $1.83 E-05$ & $7.46 E-09$ & -3.389144 \\
\hline CMMX & 1 & $2.75 E-08$ & $1.12 E-09$ & -1.38783 \\
\hline CMNN & 2643 & $7.26 E-05$ & $9.72 E-05$ & 0.12663 \\
\hline CMNO & 7243 & 0.000199 & 0.000218 & 0.0402 \\
\hline CMNS & 2610 & $7.17 E-05$ & $5.72 E-06$ & -1.098444 \\
\hline CMNX & 30 & $8.24 E-07$ & $8.62 E-07$ & 0.01957 \\
\hline CMOO & 9551 & 0.000262 & 0.000123 & -0.33061 \\
\hline CMOS & 1041 & $2.86 E-05$ & $6.42 E-06$ & -0.648899 \\
\hline CMOX & 77 & $2.12 E-06$ & $9.68 E-07$ & -0.339447 \\
\hline CMSS & 2052 & $5.64 E-05$ & $8.40 E-08$ & -2.826573 \\
\hline CMSX & 13 & $3.57 E-07$ & $2.53 E-08$ & -1.148817 \\
\hline CMXX & 6 & $1.65 E-07$ & $1.91 E-09$ & -1.935563 \\
\hline CNNN & 122810 & 0.003373 & 0.012414 & 0.56586 \\
\hline $\mathrm{CNNO}$ & 2117811 & 0.058171 & 0.041821 & -0.143315 \\
\hline CNNS & 16884 & 0.000464 & 0.001095 & 0.37318 \\
\hline CNNX & 631 & $1.73 E-05$ & 0.000165 & 0.97912 \\
\hline $\mathrm{CNOO}$ & 2981894 & 0.081906 & 0.046962 & -0.241565 \\
\hline CNOS & 99630 & 0.002737 & 0.00246 & -0.04635 \\
\hline CNOX & 2400 & $6.59 E-05$ & 0.000371 & 0.75032 \\
\hline CNSS & 4318 & 0.000119 & $3.22 E-05$ & -0.56619 \\
\hline CNSX & 38 & $1.04 E-06$ & $9.71 E-06$ & 0.96883 \\
\hline CNXX & 68 & $1.87 E-06$ & $7.33 E-07$ & -0.406432 \\
\hline $\mathrm{COOO}$ & 683049 & 0.018762 & 0.017579 & -0.028291 \\
\hline coos & 38976 & 0.001071 & 0.001381 & 0.11057 \\
\hline COOX & 24064 & 0.000661 & 0.000208 & -0.50151 \\
\hline coss & 4524 & 0.000124 & $3.62 E-05$ & -0.536074 \\
\hline cosx & 64 & $1.76 E-06$ & $1.09 E-05$ & 0.79279 \\
\hline COXX & 84 & $2.31 E-06$ & $8.23 E-07$ & -0.447847 \\
\hline CSSS & 320 & $8.79 E-06$ & $3.16 E-07$ & -1.44474 \\
\hline CSSX & 5 & $1.37 E-07$ & $1.43 E-07$ & 0.01705 \\
\hline CSXX & 4 & $1.10 E-07$ & $2.15 E-08$ & -0.707545 \\
\hline CXXX & 12 & $3.30 E-07$ & $1.08 E-09$ & -2.483295 \\
\hline MMMM & 83 & $2.28 E-06$ & $3.86 E-14$ & -7.771426 \\
\hline MMMN & 42 & $1.15 E-06$ & $5.92 E-11$ & -4.290048 \\
\hline MMMO & 31 & $8.51 E-07$ & $6.64 E-11$ & -4.107805 \\
\hline MMMS & 379 & $1.04 E-05$ & $1.74 E-12$ & -6.777 \\
\hline MMMX & 0 & 0 & $2.62 E-13$ & -- \\
\hline MMNN & 85 & $2.33 E-06$ & $3.40 E-08$ & -1.836638 \\
\hline MMNO & 113 & $3.10 E-06$ & $7.64 E-08$ & -1.608913 \\
\hline MMNS & 364 & $1.00 E-05$ & $2.00 E-09$ & -3.698853 \\
\hline MMNX & 0 & 0 & $3.02 E-10$ & -- \\
\hline MMOO & 320 & $8.79 E-06$ & $4.29 E-08$ & -2.311659 \\
\hline MMOS & 104 & $2.86 E-06$ & $2.25 E-09$ & -3.104429 \\
\hline MMOX & 3 & $8.24 E-08$ & $3.39 E-10$ & -2.386025 \\
\hline MMSS & 254 & $6.98 E-06$ & $2.94 E-11$ & -5.375177 \\
\hline MMSX & 2 & $5.49 E-08$ & $8.87 E-12$ & -3.791851 \\
\hline MMXX & 0 & 0 & $6.69 E-13$ & -- \\
\hline MNNN & 1048 & $2.88 E-05$ & $8.69 E-06$ & -0.520184 \\
\hline
\end{tabular}


TABLE 8: Continued.

\begin{tabular}{|c|c|c|c|c|}
\hline Quad & Count & $f_{i j k l}$ & $p_{i j k l}$ & $s_{i j k l}$ \\
\hline MNNO & 1323 & $3.63 E-05$ & $2.93 E-05$ & -0.093906 \\
\hline MNNS & 562 & $1.54 E-05$ & $7.67 E-07$ & -1.303999 \\
\hline MNNX & 6 & $1.65 E-07$ & $1.16 E-07$ & -0.153922 \\
\hline MNOO & 4193 & 0.000115 & $3.29 E-05$ & -0.544515 \\
\hline MNOS & 352 & $9.67 E-06$ & $1.72 E-06$ & -0.74942 \\
\hline MNOX & 31 & $8.51 E-07$ & $2.60 E-07$ & -0.515747 \\
\hline MNSS & 793 & $2.18 E-05$ & $2.25 E-08$ & -2.985098 \\
\hline MNSX & 5 & $1.37 E-07$ & $6.80 E-09$ & -1.305273 \\
\hline MNXX & 9 & $2.47 E-07$ & $5.13 E-10$ & -2.683083 \\
\hline MOOO & 5790 & 0.000159 & $1.23 E-05$ & -1.111435 \\
\hline MOOS & 167 & $4.59 E-06$ & $9.67 E-07$ & -0.676269 \\
\hline MOOX & 171 & $4.70 E-06$ & $1.46 E-07$ & -1.508056 \\
\hline MOSS & 211 & $5.80 E-06$ & $2.53 E-08$ & -2.359752 \\
\hline MOSX & 4 & $1.10 E-07$ & $7.64 E-09$ & -1.158007 \\
\hline MOXX & 55 & $1.51 E-06$ & $5.76 E-10$ & -3.418848 \\
\hline MSSS & 62 & $1.70 E-06$ & $2.21 E-10$ & -3.8869 \\
\hline MSSX & 2 & $5.49 E-08$ & $1.00 E-10$ & -2.739925 \\
\hline MSXX & 0 & 0 & $1.51 E-11$ & -- \\
\hline MXXX & 16 & $4.39 E-07$ & $7.58 E-13$ & -5.763152 \\
\hline NNNN & 5639 & 0.000155 & 0.000833 & 0.7304 \\
\hline NNNO & 60175 & 0.001653 & 0.00374 & 0.35461 \\
\hline NNNS & 538 & $1.48 E-05$ & $9.79 E-05$ & 0.82132 \\
\hline NNNX & 39 & $1.07 E-06$ & $1.48 E-05$ & 1.13953 \\
\hline NNOO & 384854 & 0.010571 & 0.006299 & -0.224828 \\
\hline NNOS & 6209 & 0.000171 & 0.00033 & 0.28656 \\
\hline NNOX & 354 & $9.72 E-06$ & $4.98 E-05$ & 0.70907 \\
\hline NNSS & 319 & $8.76 E-06$ & $4.32 E-06$ & -0.307157 \\
\hline NNSX & 6 & $1.65 E-07$ & $1.30 E-06$ & 0.898 \\
\hline NNXX & 7 & $1.92 E-07$ & $9.83 E-08$ & -0.29148 \\
\hline NOOO & 227156 & 0.006239 & 0.004716 & -0.121592 \\
\hline NOOS & 11871 & 0.000326 & 0.00037 & 0.05545 \\
\hline NOOX & 3214 & $8.83 E-05$ & $5.59 E-05$ & -0.198618 \\
\hline NOSS & 951 & $2.61 E-05$ & $9.70 E-06$ & -0.430162 \\
\hline NOSX & 13 & $3.57 E-07$ & $2.93 E-06$ & 0.9136 \\
\hline NOXX & 66 & $1.81 E-06$ & $2.21 E-07$ & -0.914541 \\
\hline NSSS & 35 & $9.61 E-07$ & $8.47 E-08$ & -1.055088 \\
\hline NSSX & 0 & 0 & $3.83 E-08$ & -- \\
\hline NSXX & 0 & 0 & $5.78 E-09$ & -- \\
\hline NXXX & 3 & $8.24 E-08$ & $2.91 E-10$ & -2.452665 \\
\hline OOOO & 61473 & 0.001689 & 0.001324 & -0.105657 \\
\hline oOOS & 5019 & 0.000138 & 0.000139 & 0.00255 \\
\hline OOOX & 9614 & 0.000264 & $2.09 E-05$ & -1.101242 \\
\hline OOSS & 331 & $9.09 E-06$ & $5.45 E-06$ & -0.222484 \\
\hline oosx & 45 & $1.24 E-06$ & $1.64 E-06$ & 0.12365 \\
\hline OOXX & 144 & $3.96 E-06$ & $1.24 E-07$ & -1.504034 \\
\hline OSSS & 38 & $1.04 E-06$ & $9.51 E-08$ & -1.040448 \\
\hline OsSX & 3 & $8.24 E-08$ & $4.30 E-08$ & -0.282172 \\
\hline OSXX & 0 & 0 & $6.49 E-09$ & -- \\
\hline OXXX & 5 & $1.37 E-07$ & $3.26 E-10$ & -2.624158 \\
\hline SSSS & 11 & $3.02 E-07$ & $6.23 E-10$ & -2.686034 \\
\hline SSSX & 0 & 0 & $3.76 E-10$ & -- \\
\hline SSXX & 0 & 0 & $8.50 E-11$ & -- \\
\hline SXXX & 0 & 0 & $8.55 E-12$ & -- \\
\hline XXXX & 0 & 0 & $3.22 E-13$ & -- \\
\hline
\end{tabular}




\section{References}

[1] M. Masso, "All-atom four-body knowledge-based statistical potentials to distinguish native protein structures from nonnative folds," BioMed Research International, vol. 2017, Article ID 5760612, 17 pages, 2017. 


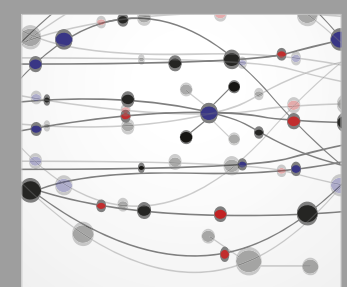

The Scientific World Journal
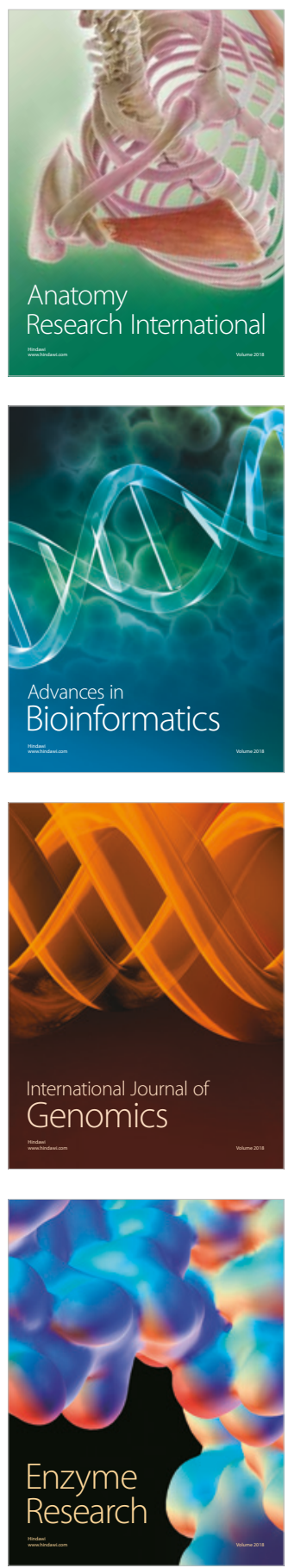
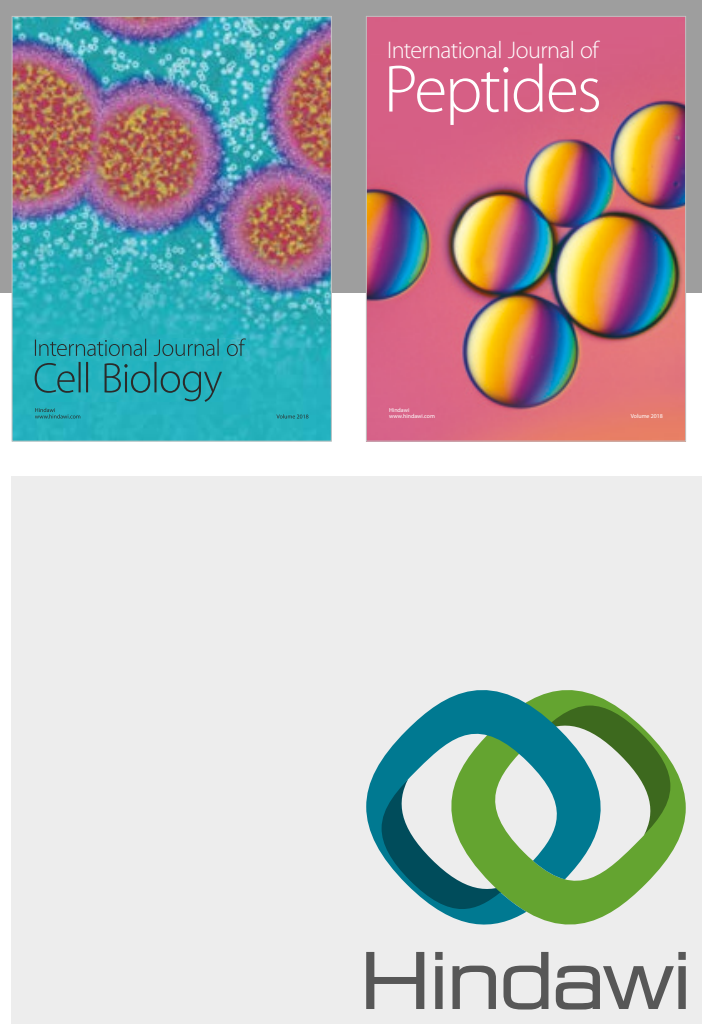

Submit your manuscripts at

www.hindawi.com
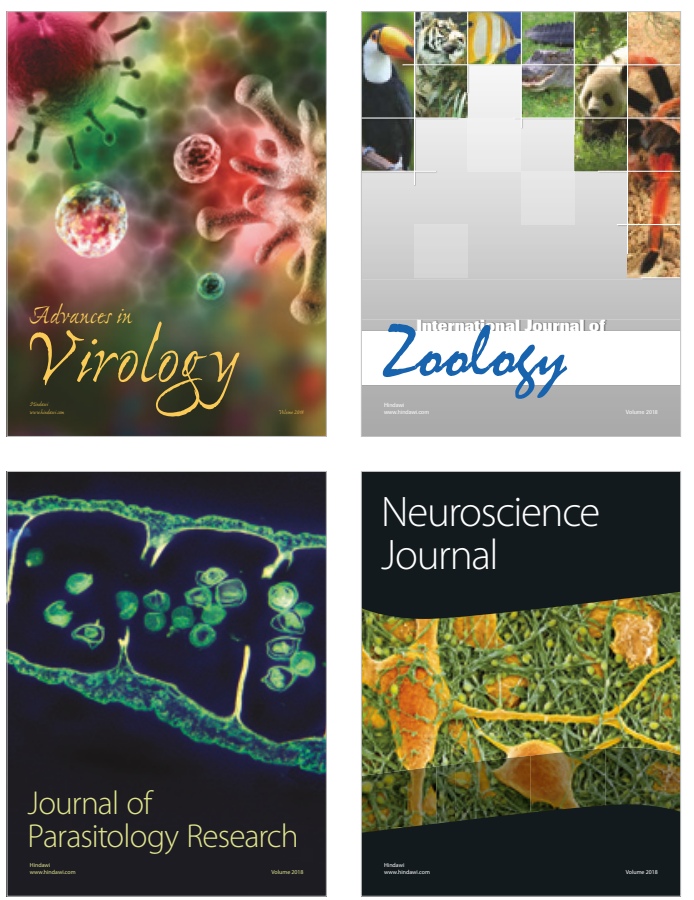
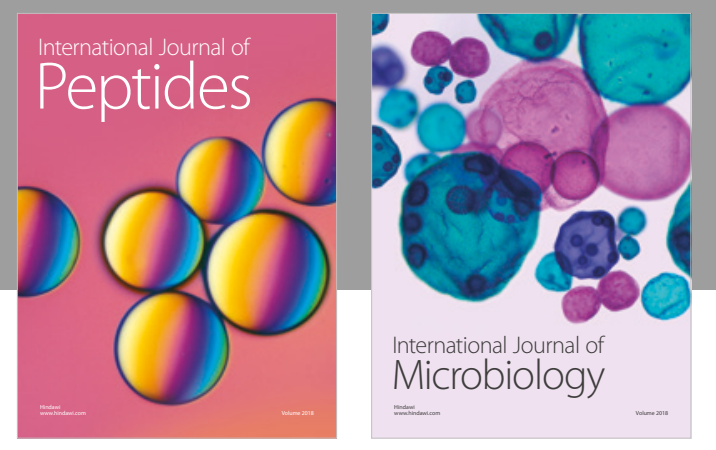

nternational Journal of Microbiology
Journal of
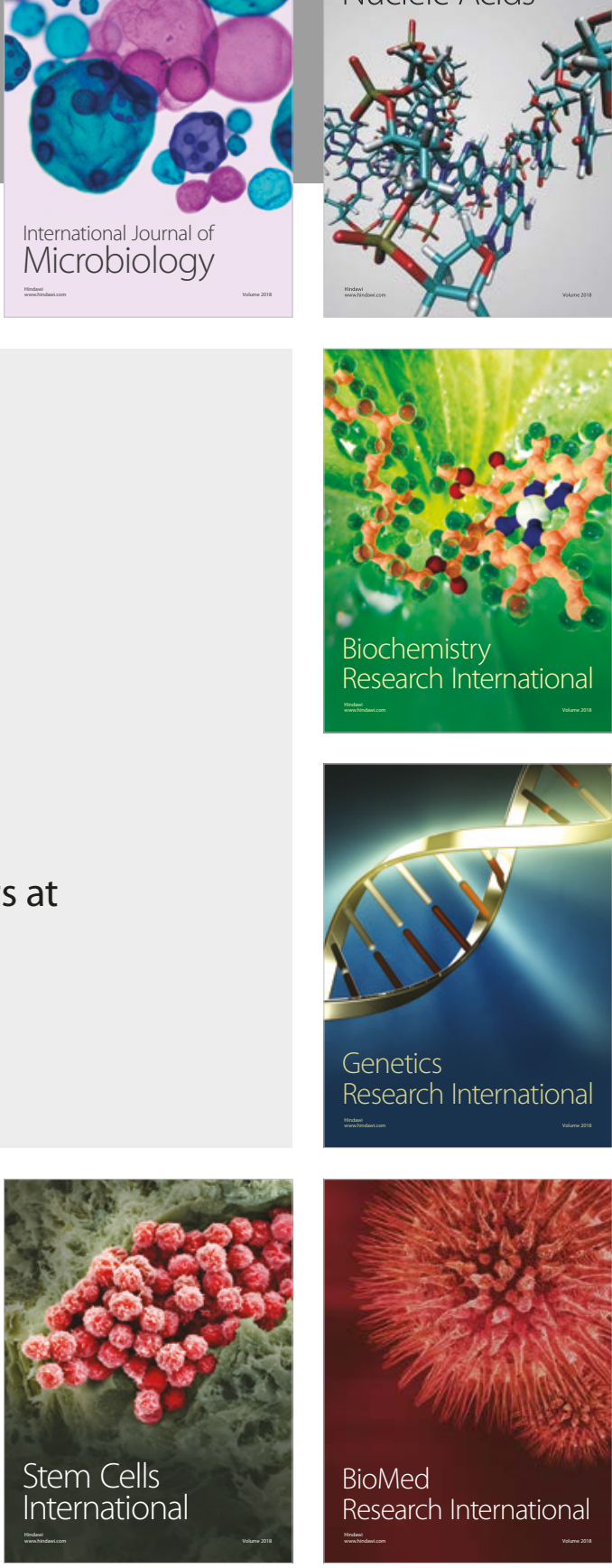
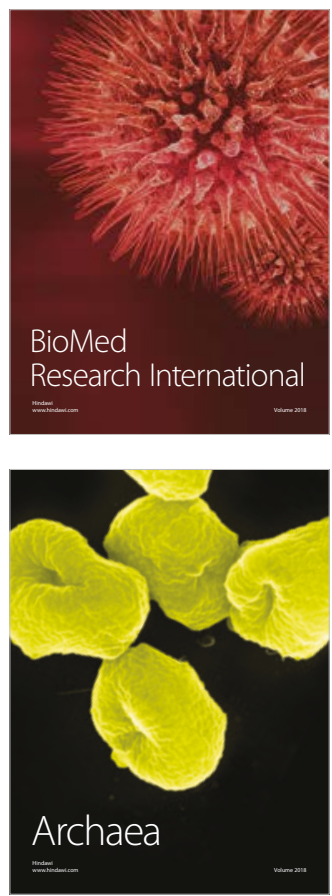